\title{
Floxuridine and Its Influence on Postnatal Cerebellar Development
}

\author{
Jan Langman ${ }^{381}$, Morimi Shimada, and Patricia Rodier \\ Department of Anatomy, School of Medicine, University of Virginia, Charlottesville, Virginia, USA
}

\begin{abstract}
Extract.
Human infants with cytomegalovirus and herpes simplex are presently treated with floxuridine (5-FUDR), a DNA synthesis blocking agent. Since in the cerebellum of the newborn infant neuron formation continues for some time after birth, the question arises whether 5-FUDR might cause permanent damage to the cerebellum. In our experiment 21 2-day-old mice were treated by three injections of 5-FUDR $(50 \mathrm{mg} / \mathrm{kg}$ body weight per injection), and particular attention was given to damage and subsequent repair of the external granular layer, the site of postnatal neuron formation. In the anterior lobes of the vermis most proliferating external granular cells died and repair was minimal. Purkinje cells were dispersed and few if any basket cells were formed. In the posterior lobes, including the uvula, considerable repair of the external granular layer was noted. The final cerebellar architecture, however, was never normal, and heterotopic granule cell nests were found in the molecular layer. In the intermediary lobes repair occurred, but Purkinje cells were found throughout the granular layer. Since the human cerebellum at birth is characterized by a thick external granular layer, it is not impossible that 5-FUDR may also cause disturbances in the human cerebellar architecture and function.
\end{abstract}

\section{Speculation}

In the cerebellum of the newborn mouse and newborn infant important neurons are formed after birth. Therapy with drugs interfering with DNA, RNA, or protein synthesis should therefore be avoided. Although repair may occur in the gastrointestinal tract, experiments with mice suggest that repair of damage in the cerebellum is minimal. Whether ectopic cell nests in the molecular layer will start cell proliferation during further life is presently not known.

\section{Introduction}

During the last 10 years 5-fluoro-2'-deoxyuridine (5FUDR), also known as floxuridine, has been used in the treatment of acute childhood leukemia [16], neuroblastoma, Hodgkin's disease [35], and a variety of other tumors such as breast and rectal carcinoma [9, 12]. Melanomas and other advanced tumors similarly have been treated with 5-iodo-2'-deoxyuridine (5IUDR), a closely related compound $[4,5]$. Both drugs were used because they inhibit the activity of the enzyme thymidylate synthetase, thereby blocking DNA replication [7, 26, 27, 32].

More recently 5-FUDR and 5-IUDR have been used as antiviral agents. Evans et al. [10] treated an 8-yearold patient with herpes simplex encephalitis with 
IUDR, while Cangir et al. [6] demonstrated the effectiveness of FUDR in children with leukemia and cytomegalovirus pneumonia. Conchie et al. [8] observed encouraging results after idoxuridine treatment of a 14-week infant with congenital cytomegalovirus. Tuffli and Nahmias [33] gave IUDR to two premature infants with herpes simplex. One infant died, while the other recovered without significant damage. In 1971 Feigin et al. [11] reported the FUDR treatment $(0.5$ $\mathrm{mg} / \mathrm{kg}$ ) of a 12-day-old infant with cytomegalic inclusion disease. Although little success could be claimed, it was recommended that consideration should be given to higher doses and prolonged duration of FUDR treatment.

Since presently very young infants are being treated with FUDR and IUDR, the question arises whether the antiviral therapy may cause dlamage of a permanent nature. While it is known that temporary suppression of DNA synthesis causes damage to all proliferating cell populations, usually this damage is followed by repair as soon as the treatment is terminated. Repair as observed in the gastrointestinal and urinary tracts, however, may not occur if the damage involves the central nervous system.

Neuron formation in the brain stem and cerebral hemispheres of humans is almost entirely finished before birth, but in the cerebellum it continues for a considerable period after birth. The site for this postnatal neuron formation is in the external granular layer, which reaches its maximum thickness shortly after birth $[28,29]$. The present study was undertaken to examine the effect of 5-FUDR on the external granular layer of the mouse shortly after birth. Particular attention was given to the repair mechanisms of this layer in the various lobes of the vermis.

\section{Materials and Methods}

Twenty-one 2-day-old suckling mice (DUB/ICR strain) were injected intraperitoneally with a saline solution of 5 -FUDR. Three injections $(50 \mathrm{mg} / \mathrm{kg}$ body weight per injection) were given at 6 -hr intervals. The dose selected was below the toxic level [17]. The controls received the same treatment as the experimental animals but without 5 -FUDR. The animals were sacrificed $3,4,6,8,10,15,20$, and 30 days after birth.

Brains of the experimental and control animals were fixed in either Bouin's or Carnoy's fixative for $24 \mathrm{hr}$. They were embedded in paraffin and 6- to 8- $\mu$ sagittal sections were cut through the vermis. The sections were stained with hematoxylin and eosin or with $1 \%$ cresyl violet.

To determine the sites of DNA synthesis at the time of the FUDR treatment, several 2-day-old mice were injected intraperitoneally with two doses of tritiated thymidine $(6 \mu \mathrm{Ci} / \mathrm{g}$ body weight) with a 4 -hr interval between injections. The animals were sacrificed $1 \mathrm{hr}$ after the last injection. The brains were fixed in cacodylate-buffered glutaraldehyde for $2 \mathrm{hr}$ [18] and washed in diluted cacodylate buffer plus $\mathrm{CaCl}_{2}$ for 24 hr. The tissues were embedded in plastic and cut at 1 $\mu$. The sections were coated with emulsion $[19,36]$. Subsequently, they were exposed for 3 weeks, developed, and mounted according to the usual histological techniques.

In this paper the ventral and clorsal lobules of the lobulus centralis and culmen are referred to as the anterior lobes of the vermis. Similarly the declive and tuber are referred to as the intermediate lobes, and the pyramis, uvula, and nodulus as the posterior lobes.

\section{Results}

During the first few days after birth the cerebellum of the mouse is characterized by an external granular layer, consisting of four to seven cell rows (Fig. 1). The cells adjacent to the pia have a large nucleus; those located more centrally have a much smaller nucleus. When labeled with tritiated thymidine, most of the large nuclei bordering the pia become radioactive, while the smaller cells fail to incorporate the label (Fig. 1). Hence the large cells are synthesizing DNA; the small ones fail to do so. The Purkinje cells similarly fail to incorporate the label, although a few cells in the (internal) granular layer synthesize DNA.

Figure 2 shows the external granular layer of a 3day-old mouse treated with three successive doses of 5 -FUDR $(50 \mathrm{mg} / \mathrm{kg}$ body weight) on day 2 and killed $12 \mathrm{hr}$ after the last injection. Many degenerating cells are present in the external granular layer. The cell degeneration is not restricted to the external granular layer but is also seen in the (internal) granular layer.

Twenty-four to $48 \mathrm{hr}$ after treatment the degenerating cells have disappeared, and only a few surviving cells are found. In the anterior regions, where destruction of the external granular layer is most extensive, the Purkinje cells seem to be dispersed in the molecular layer. By day 8 after birth, when in the controls the external granular layer is $7-10$ cells thick and the Purkinje cells are arranged in a regular row (Fig. 3), 

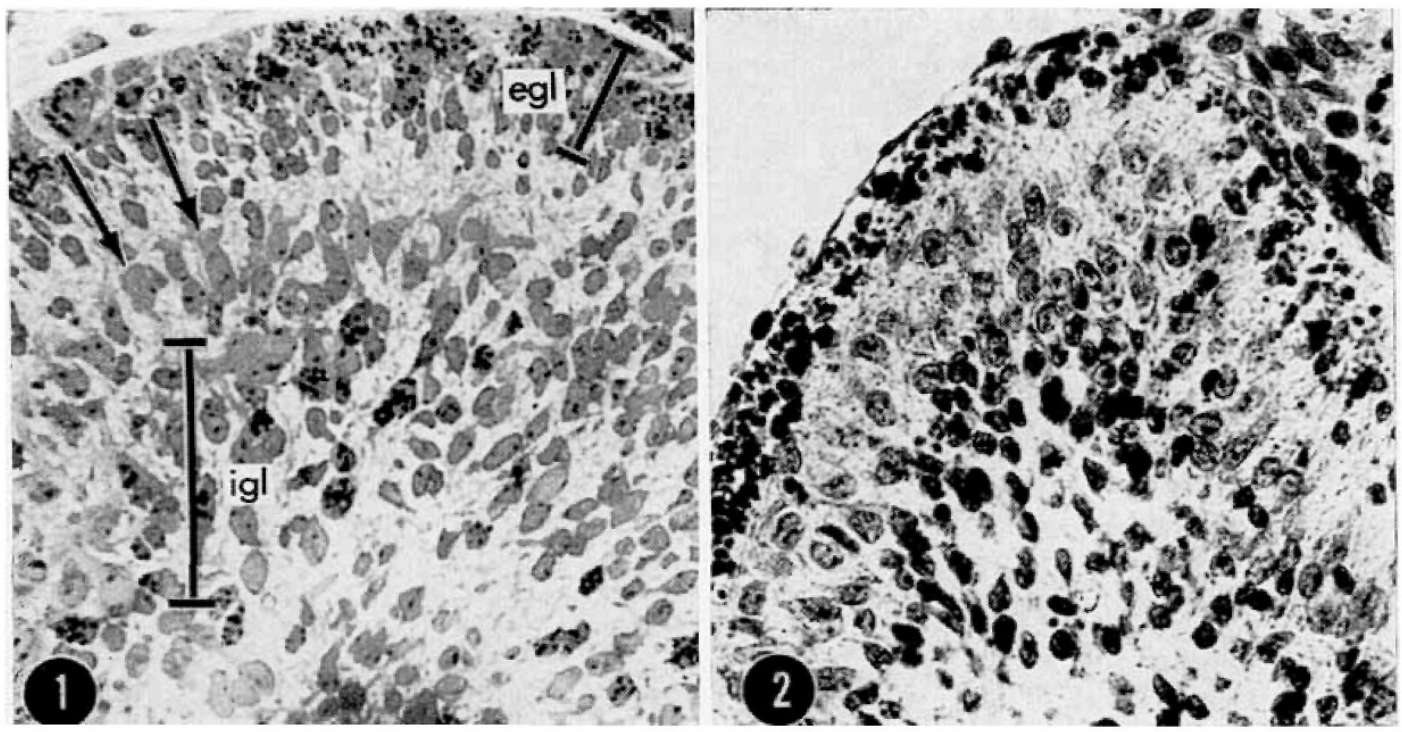

Fig. 1. Radioautograph of a $1-\mu$ section through the external granular layer $(e g l)$ of a 2-day-old mouse. Most of the nuclei of the cells in the subpial position are labeled; the more centrally located small cells are not. The Purkinje cells (arrows) are unlabeled, but a few cells in the internal granular layer $(\mathrm{igl})$ are labeled. $\times 400$.

Fig. 2. Section through an anterior lobe of the cerebellum of a 3-day-old mouse treated with three successive injections of 5-FUDR on day 2. Note the degenerating cells and nuclear debris in the external granular layer. Several cells in the internal granular layer also show degeneration. $\times 250$.

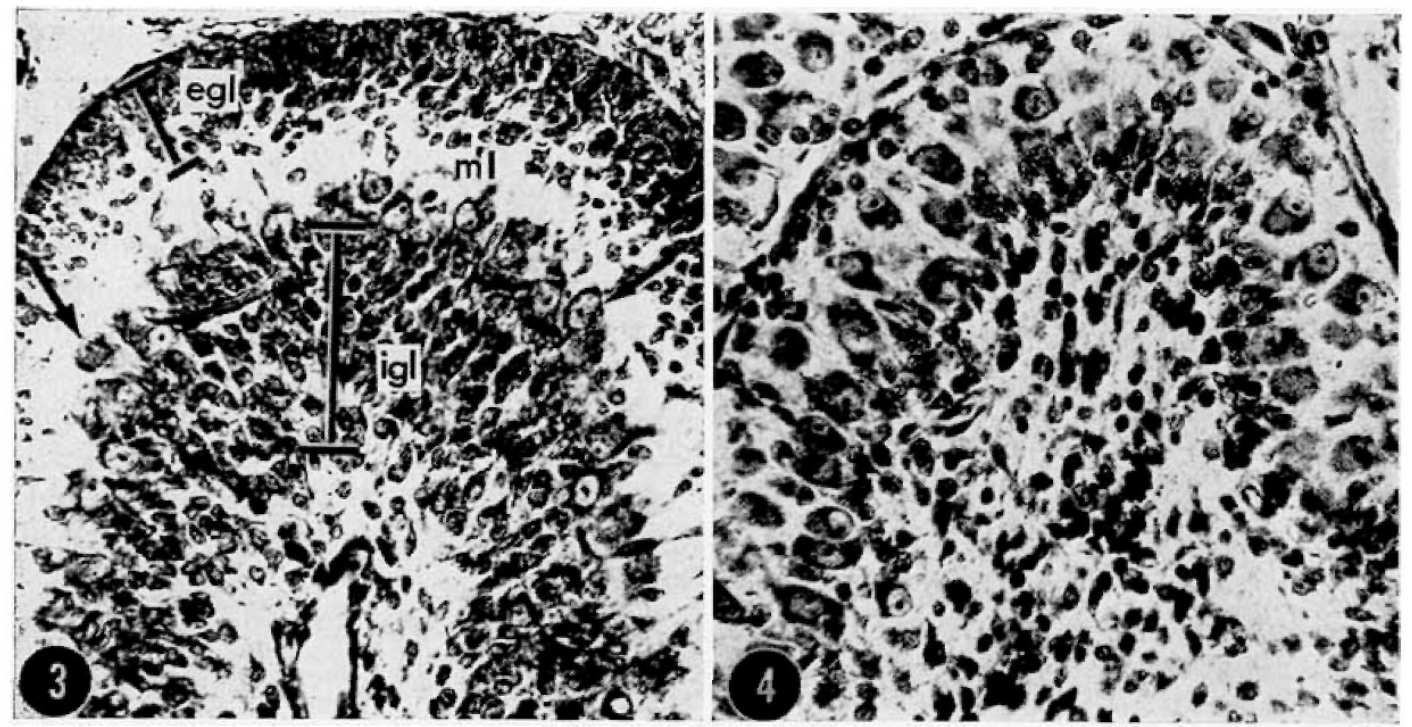

Fig. 3. Portion of an anterior lobe of the cerebellum of an 8-day-old control mouse. Note the external granular layer (egl), molecular layer $(m l)$, Purkinje cells (arrows), and internal granular layer $(i g l) . \times 250$.

Fig. 4. Section through the anterior lobe of the cerebellum of an 8-day-old mouse, treated as in Figure 2. The external granular layer is almost completely absent; the Purkinje cells are dispersed throughout the molecular layer, and several have a cytoplasmic cone extending centrally. The internal granular layer is less densely populated than that of the control. $\times 250$.

the external granular layer of the treated animals is almost entirely absent and the Purkinje cells are dispersed throughout the molecular layer (Fig. 4). In addition, many Purkinje cells have a cytoplasmic cone extending centrally instead of toward the pial surface. By day 20 , when the controls have a cerebellum characteristic of the adult, histologically the treated animals still resemble the experimental animals on day 8 . 


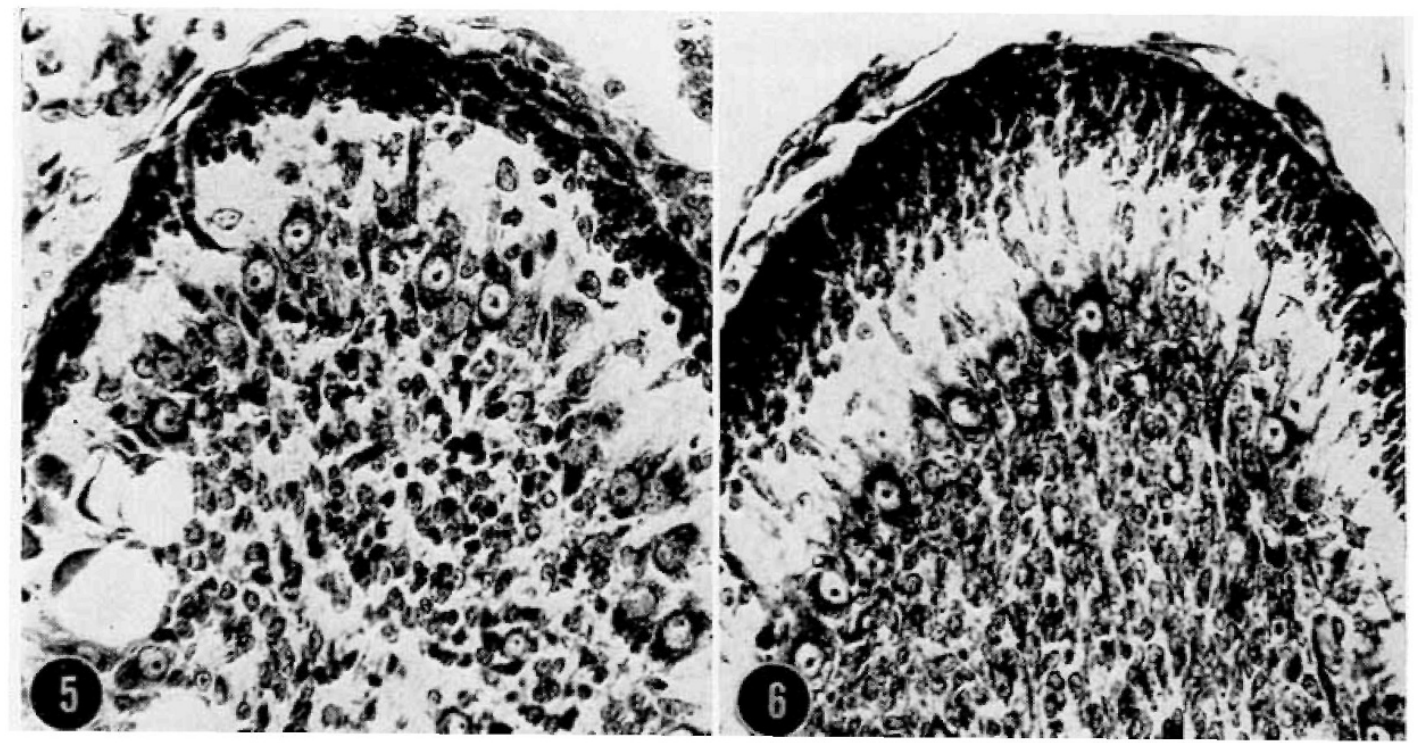

Fig. 5. Section through the uvula of an 8-day-old mouse, treated with 5-FUDR on day 2. The external granular layer consists of three to four densely packed cell layers. The Purkinje cells, contrary to those in the anterior lobes, occupy a normal position. $\times 250$. Fig. 6. Sagittal section through the uvula of an 8-day-old control mouse. Note the external granular layer, the molecular layer and the position of the Purkinje celis. $\times 250$.

Hence, repair in the anterior lobes of the cerebellum is minimal or nonexistent. Since under normal conditions the external granular layer would have produced basket, stellate, and granular neurons, it must be concluded that in the experimental animals these cells have not been formed.

Initially the histologic picture of the posterior lobes is not much different from that of the anterior lobes. By clay 6 , however, the surviving cells of the external granular layer have started to proliferate and by day 8 a tightly packed layer of cells is visible along the pial surface (Fig. 5). This new external granular layer is considerably thinner than that of the control (Fig. 6). In the 2nd week after treatment with FUDR some of the external granular cells migrate centrally. By the end of the 3rd week, however, when in the controls the external granular layer has completely disappeared, the molecular layer of the treated animals still contains many granule cells (Fig. 7). These heterotopic cells have the appearance of granule cells but they subsequently fail to migrate centrally. Hence, considerable repair occurs in the external granular layer of the posterior lobes, but this repair results in the formation of ectopic cell nests.

The architecture of the intermediate lobes of the treated animals is different from that in the anterior and posterior lobes. Although by day 20 a narrow molecular layer is present, the Purkinje cells are widely dispersed and separated from each other by large numbers of light and dark nucleated cells (Fig. 8).

\section{Discussion}

It is evident that FUDR treatment of 2-day-old mice causes extensive damage to the external granular layer. This is not surprising as most of the cells in the outer zone of the external granular layer are synthesizing DNA during the treatment (Fig. 1).

Despite the high closes of 5-FUDR given in three successive injections, a number of external granular cells always seemed to escape damage. This number was usually low in the anterior lobes, but always higher in the posterior lobes. This observation is in agreement with that of Altman et al. [3], who studied the effect of postnatal $x$-irradiation on the cerebellum.

To accomplish repair and to compensate for the cells lost during treatment, the surviving cells must undergo a number of extra divisions. No indication of such compensatory proliferation was found in the anterior lobes, and basket and stellate neurons (normally formed by the external granule cells in the 1st and 2nd week $[3,13,22])$ could not be detected. The Purkinje cells, formed early during intrauterine life, became disoriented and crowded in the space between the pial surface and the (internal) granular layer. Similar observations were made after postnatal x-irradiation $[2$, 


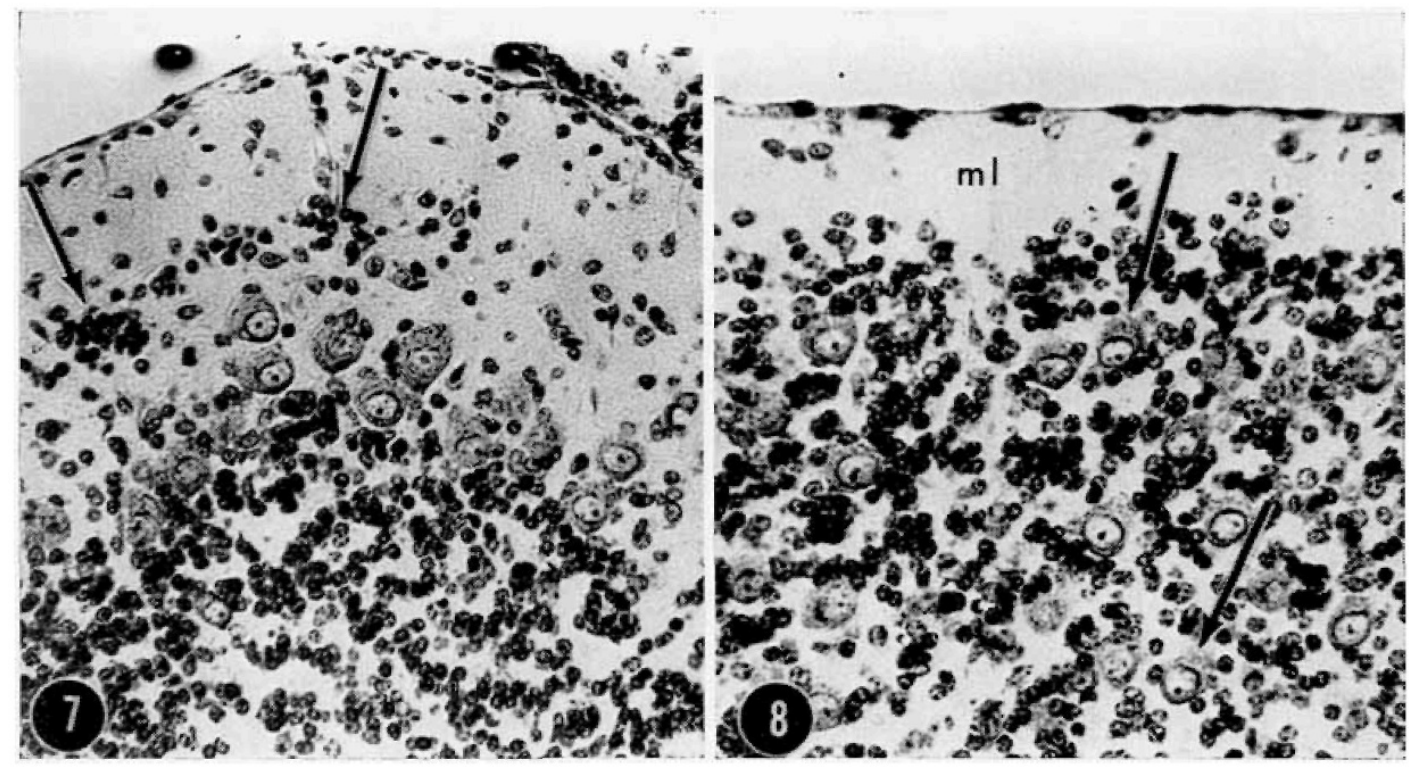

Fig. 7. Section through the uvula of a 20-day-old mouse treated on day 2. Clusters and rows of granule cells are found in a heterotopic position within the molecular layer (arrows). The Purkinje cells are in a normal position. $X 250$.

Fig. 8. Section through an intermediate lobe of the cerebellum of a 30-day-old mouse treated on day 2. A thin molecular layer ( $\mathrm{ml}$ ) is present. The Purkinje cells (arrows) are widely dispersed and separated from each other by dark and light nucleated cells. $\times 300$.

3] and after postnatal treatment with methylazoxymethanol [30].

In the posterior lobes considerable repair occurred in the 1st week after treatment, but the new external granular layer never reached the same thickness as that in the controls. Many cells failed to reach their final destination and became located in the molecular layer, where they formed ectopic cell nests. These abnormalities have also been seen after postnatal treatment with cyclophosphamide [23] and after postnatal $\mathrm{x}$-irradiation of mice, rats, and dogs $[1-3,24,25]$.

In the intermediate lobes the external granular layer showed considerable repair, but the Purkinje cells were widely dispersed in the internal granular layer, a histologic picture somewhat similar to that described for the mutant mouse "reeler" $[14,15,21]$.

The results of our experiments raise two important questions about the use of FUDR in human infants: (I) does the drug have the same effect on DNA-synthesizing cells in humans as in animals; and (2) what is the stage of cerebellum development in infants during the lst postnatal month? The biologic ability of FUDR to inhibit the action of thymidylate synthetase and the observation that the drug temporarily suppresses the proliferation of hematopoietic cells in humans seem to indicate that it will interfere with cell proliferation in general and thus probably with that in the cerebellum. With regard to the developmental stage of the human cerebellum at birth Raaf and Kernohan [28] reported that the external granular layer reaches its maximum thickness during the 1st postnatal month. During the following months it gradually decreases in thickness, and by the end of the 1st postnatal year it has disappeared. This observation was recently confirmed by Rakic and Sidman [29]. These authors also found that some daughter cells of the external granular layer migrated centrally by the 16 th week of development and that by the 32 nd week some of these cells had differentiated into basket cells. Although in the mouse, rat, and hamster the basket, stellate, and granule neurons originate from the external granular layer after birth, in the human fetus apparently some of them are formed before birth. Since, however, in the human infant the external granular layer reaches its maximum thickness after birth, it seems probable that many new neurons will be formed during the 1st postnatal year. Consequently, although there is no solid evidence that FUDR given to human infants will damage the external granular layer and result either in a decrease in the number of neurons or in abnormal repair patterns, this study certainly points to the danger.

From the few cases thus far reported in the literature, it is difficult to determine whether the neurologic deficits observed in treated infants $[8,11,33]$ are exclusively caused by the effect of the virus or are due, in 
part, to the drug therapy. Many of the symptoms observed in treated infants are similar to those observed in untreated cases of cytomegalovirus [20, 31, 34]. Keeping in mind, however, the developmental stage of the cerebellum at birth and the DNA-blocking activity of FUDR, it seems advisable to be extremely cautious in the use of floxuridine for treatment of virus diseases in newborn infants.

\section{Summary}

Since in recent years a number of human infants with cytomegalovirus and herpes simplex have been treated with 5-FUDR, a DNA synthesis-blocking agent, the question arose whether this drug might cause any permanent damage to the cerebellum. To study the effect of 5-FUDR on the cerebellum, newborn mice were treated with the drug and sacrificed at various intervals after treatment. In the anterior lobes of the vermis most proliferating cells of the external granular layer died and repair was minimal. Purkinje cells were not damaged but dispersed. Few basket cells were formed. In the posterior lobes considerable repair occurred in the external granular layer. The cerebellar architecture, however, was abnormal and heterotopic cell nests were found in the molecular layer. In the intermediary lobes repair occurred, but Purkinje cells were found throughout the granular layer. Since the human cerebellum has a substantial external granular layer at birth, it is not impossible that 5-FUDR may also cause cerebellar abnormalities when given shortly after birth.

\section{References and Notes}

1. Altman, J., Anderson, W. J., and Wright, K. A.: Selective destruction of precursors of microneurons of the cerebellar cortex with fractionated low-dose x-rays. Exp. Neurol., 17:481 (1967).

2. Altman, J., Anderson, W. J., and Wright, K. A.: Gross morphological consequences of irradiation of the cerebellum in infant rats with repsated doses of low-dose x-ray. Exp. Neurol., 21: 69 (1968).

3. Altman, J., Anderson, W. J., Anid Wright, K. A.: Reconstitution of the external granular layer of the cerebellar cortex in infant rats after low level x-irradiation. Anat. Rec., 163: 453 (1969).

4. Calabresi, P.: Current status of clinical investigations with 6azauridine, 5-iodo-2'-deoxyuridine, and related derivatives. Cancer Res., 23: 1260 (1963\}.

5. Calabresi, P., Cardoso, S. S., Finch, S. G., Kligerman, M. M., von Essen, C. F., ChU, M. Y., And Wel.ch, A. D.: Initial clinical studies with 5-iodo-2'-deoxyuridine. Cancer Res., 21: 550 (1961).
6. Cangik, A., Sullivan, M. P., Sutow, W. W., and Taylor, G.: Cytomegalovirus syndrome in children with acute leukemia. J. Amer. Med. Ass., 201: 612 (1967).

7. Cohen, S. S., Flaks, J. C., Barner, H. D., Loeb, M. R., and Lichenstein, J.: The mode of action of 5-fluorouracil and its derivatives. Proc. Nat. Acad. Sci. U.S.A., ft: 1004 (1958).

8. Conchie, A. F., Barton, B. W., and Tobin, J. O'H.: Congenital cytomegalovirus infection treated with idoxuridine. Brit. Med. J., $f: 162$ (1968).

9. Curreri, A. R., AND ANsfield, F.: Toxicity and preliminary clinical studies with 5-fluoro-2'-deoxyuridine. Cancer Chemother. Rep., 2: 8 (1959).

10. Evans, A. D., Gray, O. P., Miller, M. H., Verrier Jones, E. R., Weeks, R. D., and Wells, C. E. C.: Herpes simplex encephalitis treated with intravenous idoxyridine. Brit. Med. J., 2: 407 (1967).

I1. Feigin, R. D., Shakelford, P. G., DeVivo, D. C., and HayMOND, M. W.: Floxuridine treatment of congenital cytomegalic inclusion disease. Pediatrics, 47:318 (1971).

12. Fennelly, J. J., and Fitzgran.d, M. X.: The value of fluorinated pyrimidines in advanced malignancy. Brit. J. Cancer, 22: 673 (1968).

13. FujrTa, S.: Quantitative analysis of cell proliferation and differentiation in the cortex of the postnatal mouse cerebellum. J. Ccll Biol., 32: 277 (196ૉ).

14. Hamburgh, M.: Observations on the neuropathology of "reeler," a neurological mutation in mice. Separatum Experientia, 16: 460 (1960).

15. Hamburgh, M.: Analysis of the postnatal developmental effects of "reeler," a neurological mutation in mice. A study in developmental genetics. Develop. Biol., 8 : 165 (1963).

i6. Hartman, J. R., Origenes, M. L., Murphy, M. L., Sitarz, A., AND ERLANDSON, M.: Effects of 2'-deoxy-5-fluorouridine and 5fluorouracil on childhood leukemia. Cancer Chemother. Rep., 34: 51 (1964).

17. Heidelberger, C., Griesback, L., Cruz, O., Schnitzer, R., and Grunberg, E.: Fluorinated pyrimidine. VI. Effects of 5-fluorouridine and 5-fluoro- 2 -deoxyuridine on transplanted tumors. Soc. Exp. Biol. Med., 97: 470 (1958).

18. KaknovsKY, M. J.: A formaldehyde glutaraldehyde fixative of high osmolality for use in electron microscopy. J. Cell Biol., 27: 137 (1965).

19. Kopriws, B., ANd Leblond, C. P.: Improvements in the coating technique of radioaulography. J. Histochem. Cytochem., 10: 269 (1962).

20. Mendearis, D. N.: Observations concerning human cytomegalovirus infection and disease. Bull. Johns Hopkins Hosp., 114: 18I (1964).

21. Meier, H., and Hong, W. G.: The ncuropathology of "reeler," a neuromuscular mutation in mice. J. Neuropath. Exp. Neurol., 21: 649 (1962).

22. Mrale, I. L., And Sidman, R. L.: An autoradiographic analysis of histogenesis in the mouse cerebellum. Exp. Neurol., 4: 277 (1961).

23. Nathanson, N., Cole, G. A., and van der Loos, H.: Heterotopic cercbellar granule cells following administration of cyclophosphamide to suckling rats. Brain Res., 15: 532 (1969).

24. Phemister, R. D., Shively, J. N., and Young, S.: The effects of gamma irradiation on the postnatally developing canine ccrebellar cortex. I. Effects of single sublethal exposures. J. Neuropath. Exp. Neurol., 28: 119 (1969). 
25. Phemister, R. D., Shively, J. N., and Young, S.: The effects of gamma irradiation on the postnatally developing canine cerebellar cortex. II. Sequential histogenesis of radiation-induced changes. J. Neuropath. Exp. Neurol., 28: 128 (1969).

26. Prusoff, W. H.: Synthesis and biological activities of iododeoxyuridine, an analogue of thymidine. Biochim. Biophys. Acta, 32: 295 (1959).

27. Prusoff, W. S.: Studies on the mechanism of action of 5iododeoxyuridine, an analogue of thymidine. Cancer Res., 20: $92(1960)$.

28. RAAF, J., AND Kernohan, J. W.: A study of the external granular layer in the cerebellum. Amer. J. Anat., 75: 151 (1944).

29. Rakic, P., ANd Sidman, R. L.: Histogenesis of cortical layers in human cerebellum, particularly the lamina dissecans. J. Comp. Neurol., 139: 473 (1970).

30. Shimada, M., And Langman, J.: Repair of the external granular layer of the hamster cerebellum after prenatal and postnatal administration of methylazoxymethanol. Teratology, 3 : 119 (1970).

31. STERN, H.: Isolation of cytomegalovirus and clinical manifes- tations of infection at different ages. Brit. Med. J., 1: 665 (1968).

32. Taylor, J. H., Hart, W. F., and Tung, J.: Effects of fluorodeoxyuridine on DNA replication, chromosome breakage, and reunion. Proc. Nat. Acad. Sci. U.S.A., f8: 190 (1962).

33. Tuffl, G. A., and Nahmras, A. J. Neonatal herpetic infection. Amer. J. Dis. Child., 118: 909 (1969).

34. Weller, T. H., and Hanshaw, J. B.: Virologic and clinical observations on cytomegalic inclusion disease. New Engl. J. Med., 266: 1233 (1962).

35. Wolman, I. J., And Gens, R. D.: Progress report on the use of 5 -fluoro-2'-deoxyuridine (5-FUDR) in childhood leukemias and neoplasms. Cancer Chemother. Rep., 2: 14 (1959).

36. NTB-2 emulsion, Eastman Kodak, Rochester, N. Y.

37. Supported by Grant no. NB-06188 from the National Institute of Neurological Diseases and by Grant no. R-200-66 from the United Cerebral Palsy Foundation.

38. Requests for reprints should be addressed to: JAN LANGMan, M.D., PH.D., Department of Anatomy, School of Medicine, University of Virginia, Charlottesville, Va. 22901 (USA).

39. Accepted for publication May 2, 1972. 